\title{
(6) OPEN ACCESS \\ The influence of sexual harassment on mental health among female military personnel of the Republic of Korea Armed Forces
}

\author{
Tae Kyung Kim, ${ }^{1,2} \mathrm{H}-\mathrm{C}$ Lee, ${ }^{3}$ S G Lee, ${ }^{4} \mathrm{~K}$-T Han, ${ }^{1,2}$ E-C Park ${ }^{2,5}$
}

'Department of Public Health, Graduate School, Yonsei University, Seoul, Republic of Korea

${ }^{2}$ Institute of Health Services Research, Yonsei University, Seoul, Republic of Korea ${ }^{3}$ Department of Preventive Medicine, 65th Medical Brigade, USA

${ }^{4}$ Department of Hospital Management, Graduate School of Public Health, Yonsei University, Seoul, Republic of Korea

${ }^{5}$ Department of Preventive Medicine, College of Medicine, Yonsei University, Seoul, Republic of Korea

\section{Correspondence to} Professor Eun-Cheol Park, Department of Preventive Medicine, College of Medicine, and Institute of Health Services Research, Yonsei University, 50-1 Yonsei-ro, Seodaemun-gu, Seoul 120-752, Republic of Korea; ecpark@yuhs.ac

Received 27 December 2015 Revised 28 February 2016 Accepted 5 March 2016 Published Online First 15 April 2016

\section{(a) CrossMark}

To cite: Kim TK, Lee H-C, Lee $\mathrm{SG}$, et al. $J$ R Army Med Corps 2017:163:104-110.

\section{ABSTRACT}

Introduction Reports of sexual harassment are becoming more frequent in Republic of Korea (ROK) Armed Forces. This study aimed to analyse the impact of sexual harassment on mental health among female military personnel of the ROK Armed Forces.

Methods Data from the 2014 Military Health Survey were used. Instances of sexual harassment were recorded as 'yes' or 'no'. Analysis of variance (ANOVA) was carried out to compare Kessler Psychological Distress Scale 10 (K-10) scores. Multiple logistic regression analysis was performed to identify associations between sexual harassment and $\mathrm{K}-10$ scores.

Results Among 228 female military personnel, 13 $(5.7 \%)$ individuals experienced sexual harassment. Multiple logistic regression analysis revealed that sexual harassment had a significantly negative impact on K-10 scores $(3.486, p<0.04)$. Higher $\mathrm{K}-10$ scores among individuals experiencing sexual harassment were identified in the unmarried (including never-married) group (6.761, $p<0.04)$, the short-term military service group $(12.014$, $p<0.03)$ and the group whose length of service was $<2$ years $(11.067, p<0.02)$.

Conclusions Sexual harassment has a negative impact on mental health. Factors associated with worse mental health scores included service classification and length of service. The results provide helpful information with which to develop measures for minimising the negative psychological effects from sexual harassment and promoting sexual harassment prevention policy.

\section{INTRODUCTION}

South Korean culture is deeply rooted in Confucianism, wherein obedience and respect for one's seniors are expected. In addition, male chauvinism and a householder system, which has lasted for hundreds of years, place South Korean women at comparatively lower status than men. ${ }^{1-3}$ Male dominance in Korean society has weakened with the expansion of Western culture, bolstering the social influence of women; nevertheless, deference to men remains strong.

The Republic of Korea (ROK) Armed Forces has enlisted female personnel since 1949 when 490 women were enlisted and the current number of female military personnel in the ROK Armed Forces exceeds 9200. Female cadets are also admitted to the Korean Air Force Academy since 1997, the Korean Military Academy since 1998 and the Korean Naval Academy since 1999. The first female officer was promoted to the rank of general in 2002. The Reserved Officer"s Training Corps

\section{Key messages}

- The Republic of Korea (ROK) Armed Forces have been promoting policies to increase the proportion of female military personnel.

- Sexual harassment and sexual assault in the military increased 4.5-fold in 2013 (59 cases), compared with 2010 (13 cases).

- Generally, the experience of being sexually harassed was negatively associated with female military personnel's mental health.

- The impact on mental health as a result of sexual harassment largely depended upon the marital status, the service classification and the length of service.

(2011) and the Korean Army Academy at Yeong-cheon (2015) also permit the entrance of women among their ranks. The ROK Armed Forces also actively promotes policies to increase the proportions of female military personnel and by 2020 , their goal is to increase the proportion of female officers to $7.7 \%$ and the non-commissioned officer (NCO) ratio of female military personnel to $5.5 \%{ }^{4}$

Female military personnel have several strengths, compared with male military personnel. In particular, they show expertise in the operation of advanced equipment, record keeping and various other sophisticated skills. For this reason, the importance of the female military personnel has emerged in the ROK Armed Forces.

The percentage of women in the Korean workforce was 36\% in 1963 and has increased by $16.6 \%$ since 1963 . However, inclusion of a small number of women in male-dominated groups creates an environment for potential gender discrimination, sexual harassment and sexual abuse; compared with their male counterparts, opportunities for promotion are relatively small. Moreover, most Korean women do not return to work after childbirth, which further reduces the number of women in the workforce.

Sexual harassment and sexual assault in the military increased 4.5-fold between 2010 and 2013 (13 vs 59 cases). ${ }^{5}$ In 2014, a female officer committed suicide after ongoing harassment, and the general officer was arrested for the molestation and rape of female NCOs. These reports of sexual harassment and violence in the military have created social unrest and discussions about the treatment of 
women in the military are becoming commonplace. As the issue of sexual harassment affects the honour of the military, the ROK Armed Forces have responded with various programmes for the prevention of gender-related incidents, including mandatory, annual sexual harassment prevention training for officers and NCOs. A 'one-out' system was implemented to punish perpetrators of sexual harassment and sexual assault, and a female sexual harassment counsellor was hired to help resolve complaints involving female military personnel.

Mental health is a serious problem in Korea. ${ }^{6}$ The suicide rate in Korea (38.3 per 100000 individuals) is the highest among Organisation for Economic Co-operation and Development (OECD) member countries. The suicide rate among those serving in the military is particularly concerning. According to a 2012 survey, the prevalence of depression in the entire military was $4.6 \%$, which is 2.5 -fold higher than that for the general civilian population. ${ }^{8}$ The rates of depression by branch are $7.0 \%$ for the army, 3.0\% for the navy and $2.0 \%$ for the air force.

In a previous study of female lawyers and military personnel, those who had experienced or observed sexual harassment by male superiors or colleagues reported lower overall job satisfaction and expressed a greater intention to quit than women who had not experienced or observed sexual harassment by male superiors or colleagues. ${ }^{9}$ Also, if women reported experiencing military-related sexual assault, screening indicated a twofold higher incidence of alcohol abuse. ${ }^{10}$ Another study showed that female veterans who experienced military sexual trauma exhibit higher rates of post-traumatic stress disorder (PTSD) than those women who experienced combat trauma and traumatic death. Military sexual trauma has been shown to be directly correlated with mental health. ${ }^{11}$ Also, girls or boys who acknowledged sexually harassing others were four to six times more likely to use alcohol than their non-harassing peers, ${ }^{12}$ such that the odds of a mental health diagnosis such as PTSD, other anxiety disorders, depression and substance use disorders were increased. ${ }^{13}$ A number of studies have shown consistent gender differences regarding perceptions of, and reactions to sexual harassment in the workplace. Women were more aware of the occurrence of sexual harassment than men. Sexual abuse is a significant antecedent of suicidal behaviour, particularly among women. ${ }^{13} 14$

The importance of a study on the relationship between mental health and sexual harassment among female military personnel cannot be overstated. Although many investigations have examined the relationships of sexual harassment with mental health, they have focused on members of the UK and the US Armed Forces, overseas deployments and PTSD. ${ }^{15-17}$ To our knowledge, the present study is the first to specifically examine the relationship between sexual harassment and mental health among ROK female military personnel. We also investigated the relationships between mental health and sexual harassment according to service classification and length of military service.

\section{METHODS}

\section{Study population}

The study used data from the 2014 Military Health Survey (MHS) conducted by the School of Military Medicine, which was designed to obtain military health determinants. Raw data were obtained from the Korean Military Medical School with the Dean's approval. The institutional review board of the Armed Forces Medical Command (AFMC) provided formal ethics approval for the MHS data sets. Since the 2014 MHS is an anonymous, self-administered survey, individual responses cannot be linked to specific personnel or medical records. A total of 8012 military personnel (officer, NCO or warranty officer: 3599; enlisted soldiers: 4413) were included in the data. Male military personnel were excluded from further study. Of the 283 eligible female military personnel, 55 women were excluded from the study due to missing or incomplete data. The total number of female military personnel in the study was 228 .

\section{Variables}

The dependent variable comprised scores on the Kessler Psychological Distress Scale 10 (K-10) Index. This self-report instrument consists of 10 items and is designed to measure levels of distress in clinical and population surveys. The K-10 total score ranges from 10 to 50 . It has been widely implemented and was used in the WHO World Mental Health Survey. Prior research has shown the K-10 to be an excellent screening tool for mental health disorders. ${ }^{18}$ The 2001 Victorian Population Health Survey adopted a set of cut-off scores that can be used as a guide for psychological distress screening. ${ }^{19}$ However, K-10 test validation is dependent upon the characteristics of each country. Typically, the disorder is screened by setting the optimal score to 24 points. ${ }^{20}$ In South Korea, 76\% of subjects whose K-10 scores were $>13$ points had sought psychiatric care within the past year. $^{21}$

Military personnel responded to the 10 self-administered questions using a scale ranging from 1 to 5 . In the MHS version of the K-10, all 10 questions were asked regarding the previous month. Each question has five possible responses: 'all of the time', 'most of the time', 'some of the time', 'a little of the time' or 'none of the time' (coded as 5, 4, 3, 2 and 1, respectively). Higher scores indicate greater degrees of psychological distress. $^{22}$

The variable of primary interest was 'experience of sexual harassment'. In the MHS, the question was asked regarding the military service periods. The question has two possible responses: 'yes' and 'no' (coded as 1 and 0, respectively). Sexual harassment was separated into five levels: (a) gender harassment, generalised sexist remarks and behaviour; (b) seductive behaviour, inappropriate and offensive, but essentially sanction-free, sexual advances; (c) sexual bribery, solicitation of sexual activity or other sex-linked behaviour with promise of rewards; (d) sexual coercion, pressure for sexual activity by threat of punishment and (e) sexual assault, gross sexual imposition or assault. ${ }^{23}$ Other independent variables considered in the analysis were age, education level, marital status, dependent children, military type, branch, rank, service classification, length of service, workplace, number of working hours per week, stress from immediate superior, physical activity and weight fluctuation. Branch was classified into four levels: (a) combat branch, infantry, armour, artillery, air defence, intelligence, engineering, information/communication and aviation; (b) technical service branch, ordinance, quarter master, transportation and chemical; (c) administrative support branch, adjutant general, military police, finance and troop information and education and (d) special branch, medical, judge advocate and chaplain. Rank was classified into two groups: 'officer (above O-1)' and 'noncommissioned officer (above E-5)'. Service classification was classified into three groups: (a) long-term military service, starting from excellent short-term or extended military service personnel, ROK Armed Forces guarantees $>10$ years of service conditions for each soldier; (b) extended military service, longterm military service candidates not selected as long-term military service personnel (contracts extend military service on an annual basis until selected for long-term military service) and (c) 
short-term military service, basic service period of the military service (3 years).

\section{Statistical analysis}

For group comparisons, whether or not personnel experienced sexual harassment was analysed separately. Adjustments were made for mental health, whether or not sexual harassment was experienced and the following variables: age, education level, marital status, dependent children, military type, branch, rank, service classification, length of service, workplace, number of working hours per week, stress from an immediate superior, physical activity and weight fluctuation.

Descriptive statistics showed the general characteristics of study populations by variables. To compare the average values of the K-10 according to the independent variables, analysis of variance (ANOVA) was performed. Multiple linear regression analysis was carried out to examine the association between experiences of sexual harassment and K-10 score. Subgroup analysis was performed for the following combined variables: marital status, rank, service classification and length of service. All analyses were performed using SAS software, V.9.4 (SAS Institute, Cary, North Carolina, USA).

\section{RESULTS}

The study population consisted of 228 female military personnel of the ROK Armed Forces. Thirteen (5.7\%) female military personnel reported sexual harassment. The proportion of personnel in the 25-43 years of age group (52.2\%) was slightly higher than the 19-24 years of age group (52.2\% vs $47.8 \%$, respectively). Unmarried (including never-married) personnel totalled $177(77.6 \%)$ in the study population. With regard to service classification, the study population comprised 70 women $(30.7 \%)$ with long-term military service, 91 women $(39.9 \%)$ with short-term military service and 67 women (29.4\%) with extended military service (Table 1 ).

The average K-10 score was higher for the sexual harassment group $(20.86 \pm 10.38)$ than the no sexual harassment group $(14.81$ $\pm 6.16)$. K-10 scores by age, education level, rank, length of service, workplace, stress from immediate superior, physical activity and weight fluctuation were higher for the sexual harassment group than the no sexual harassment group. However, K-10 scores by marital status (married), dependent children (yes), military type (navy, marine), branch (technical service branch, administrative support branch), service classification (long-term military service) and work hours per week $(50<$ working hours $\leq 56)$ were lower for the sexual harassment group (Table 2).

Multiple linear regression analysis of the K-10 score was conducted to investigate the relationship between mental health and sexual harassment. The mean $\mathrm{K}-10$ score was significantly higher for the sexual harassment group than the no sexual harassment group $(\beta=3.486, p=0.0397)$. Scores were significantly higher for unmarried female military personnel than married female military personnel $(\beta=3.401, p=0.0348)$. K-10 score for stress from immediate superior in the 'high' group was higher than the 'low' group $(\beta=2.592, p=0.0016$; Table 3$)$.

Subgroup analyses were performed by marital status, rank, service classification and length of service. In the unmarried group, female military personnel who experienced sexual harassment tended to have higher K-10 scores $(\beta=6.761, p=0.0355)$. In the short-term military service group, female military personnel who had experienced sexual harassment tended to have higher K-10 scores $(\beta=12.014, p=0.0269)$. In the extended military service group, female military personnel who had experienced sexual harassment tended to have higher K-10 scores $(\beta=4.666$,
Table 1 General characteristics of the study population

\begin{tabular}{|c|c|}
\hline & N (\%) \\
\hline \multicolumn{2}{|l|}{ Experience of sexual harassment } \\
\hline Yes & $13(5.7)$ \\
\hline No & $215(94.3)$ \\
\hline \multicolumn{2}{|l|}{ Age (years) } \\
\hline $19-24$ & $109(47.8)$ \\
\hline $25-43$ & $119(52.2)$ \\
\hline \multicolumn{2}{|l|}{ Education } \\
\hline$\leq$ High school & $59(25.9)$ \\
\hline$\geq$ Graduate from university & $169(74.1)$ \\
\hline \multicolumn{2}{|l|}{ Marital status } \\
\hline Married & $51(22.4)$ \\
\hline Unmarried & $177(77.6)$ \\
\hline \multicolumn{2}{|l|}{ Dependent children } \\
\hline Yes & $35(15.4)$ \\
\hline No & $193(84.7)$ \\
\hline \multicolumn{2}{|l|}{ Military type } \\
\hline Army & $150(65.8)$ \\
\hline Navy, Marine & $54(23.7)$ \\
\hline Air force & $24(10.5)$ \\
\hline \multicolumn{2}{|l|}{ Branch } \\
\hline Combat branch & $97(42.5)$ \\
\hline Technical service branch & $69(30.3)$ \\
\hline Administrative support branch & $26(11.4)$ \\
\hline Special branch & $36(15.8)$ \\
\hline \multicolumn{2}{|l|}{ Rank } \\
\hline Officer & $63(27.6)$ \\
\hline Non-commissioned officer & $165(72.4)$ \\
\hline \multicolumn{2}{|l|}{ Service classification } \\
\hline Long-term military service & $70(30.7)$ \\
\hline Short-term military service & $91(39.9)$ \\
\hline Extended military service & $67(29.4)$ \\
\hline \multicolumn{2}{|l|}{ Length of services (years) } \\
\hline$\leq 2$ & $101(44.3)$ \\
\hline$\geq 3$ & $127(55.7)$ \\
\hline \multicolumn{2}{|l|}{ Workplace } \\
\hline Urban & $101(44.3)$ \\
\hline Rural & $118(51.8)$ \\
\hline Isolated area & $9(4.0)$ \\
\hline \multicolumn{2}{|l|}{ Work hours per week } \\
\hline$\leq 40$ & $40(17.5)$ \\
\hline$\leq 48$ & $53(23.3)$ \\
\hline$\leq 56$ & $80(35.1)$ \\
\hline$\geq 57$ & $55(24.1)$ \\
\hline \multicolumn{2}{|l|}{ Stress from immediate superior } \\
\hline High & $147(64.5)$ \\
\hline Low & $81(35.5)$ \\
\hline \multicolumn{2}{|l|}{ Physical activity (days of activity per week) } \\
\hline 0 per week & $82(36.0)$ \\
\hline $1-2$ per week & $78(34.2)$ \\
\hline$\geq 3$ per week & $68(29.8)$ \\
\hline \multicolumn{2}{|l|}{ Weight fluctuation } \\
\hline Yes (loss or gain in weight $(\geq 3 \mathrm{~kg})$ ) & $142(62.3)$ \\
\hline None & $86(37.7)$ \\
\hline
\end{tabular}

$\mathrm{p}=0.0385)$. The short-term military service group was more affected by sexual harassment than the extended military service group. When female military personnel experienced sexual harassment, personnel whose length of service were $<2$ years tended to have higher K-10 scores $(\beta=11.067, p=0.0159$; Table 4). 
Table 2 Mean scores on the K-10 Index for the study population

\begin{tabular}{|c|c|c|c|c|c|c|c|c|c|}
\hline & \multirow{2}{*}{\multicolumn{3}{|c|}{ Total }} & \multicolumn{6}{|c|}{ Experience of sexual harassment } \\
\hline & & & & \multicolumn{3}{|l|}{ Yes } & \multicolumn{3}{|l|}{ No } \\
\hline & Mean & SD & $p$ Value & Mean & SD & $\mathrm{p}$ Value & Mean & SD & $\mathrm{p}$ Value \\
\hline \multicolumn{10}{|l|}{ Age (years) } \\
\hline $19-24$ & 15.20 & 6.61 & 0.7714 & 20.86 & 10.38 & 0.1712 & 14.81 & 6.16 & 0.8068 \\
\hline $25-43$ & 14.99 & 5.49 & & 15.00 & 5.51 & & 14.99 & 5.52 & \\
\hline \multicolumn{10}{|l|}{ Education } \\
\hline$\leq$ High school & 15.15 & 5.28 & 0.9213 & 15.50 & 3.54 & 0.8422 & 15.14 & 5.35 & 0.6988 \\
\hline$\geq$ Graduate from university & 15.07 & 6.30 & & 18.64 & 9.38 & & 14.82 & 5.99 & \\
\hline \multicolumn{10}{|l|}{ Marital status } \\
\hline Married & 13.35 & 2.88 & 0.0104 & 12.00 & 0.71 & 0.004 & 13.50 & 2.99 & 0.0438 \\
\hline Unmarried & 15.59 & 6.60 & & 22.00 & 9.26 & & 15.29 & 6.33 & \\
\hline \multicolumn{10}{|l|}{ Dependent children } \\
\hline Yes & 13.43 & 2.87 & 0.0511 & 12.00 & 0.00 & 0.1113 & 13.52 & 2.94 & 0.1029 \\
\hline No & 15.39 & 6.41 & & 19.27 & 9.06 & & 15.16 & 6.17 & \\
\hline \multicolumn{10}{|l|}{ Military type } \\
\hline Army & 14.96 & 5.68 & 0.0286 & 20.00 & 10.89 & 0.6370 & 14.71 & 5.24 & 0.0066 \\
\hline Navy, Marine & 17.79 & 10.35 & & 14.00 & 3.46 & & 18.33 & 10.93 & \\
\hline Air force & 14.26 & 3.89 & & 18.00 & 10.89 & & 14.04 & 3.63 & \\
\hline \multicolumn{10}{|l|}{ Branch } \\
\hline Combat branch & 15.34 & 6.69 & 0.1628 & 20.14 & 10.85 & 0.3842 & 14.97 & 6.19 & 0.1489 \\
\hline Technical service branch & 14.13 & 3.60 & & 12.00 & 0.00 & & 14.19 & 3.63 & \\
\hline Administrative support branch & 16.88 & 8.17 & & 13.00 & 0.00 & & 17.04 & 8.30 & \\
\hline Special branch & 14.97 & 6.06 & & 19.33 & 5.86 & & 14.58 & 6.01 & \\
\hline \multicolumn{10}{|l|}{ Rank } \\
\hline Officer & 15.78 & 6.32 & 0.2419 & 22.00 & 16.46 & 0.9321 & 15.47 & 5.55 & 0.3369 \\
\hline Non-commissioned officer & 14.83 & 5.93 & & 17.00 & 5.87 & & 14.69 & 5.92 & \\
\hline \multicolumn{10}{|l|}{ Service classification } \\
\hline Long-term military service & 14.23 & 4.68 & 0.0177 & 14.00 & 2.55 & 0.0708 & 14.25 & 4.82 & 0.0641 \\
\hline Short-term military service & 16.36 & 7.74 & & 29.00 & 11.53 & & 15.93 & 7.29 & \\
\hline Extended military service & 14.27 & 4.16 & & 15.80 & 6.14 & & 14.15 & 4.00 & \\
\hline \multicolumn{10}{|l|}{ Length of services (years) } \\
\hline$\leq 2$ & 15.82 & 6.59 & 0.0729 & 26.00 & 11.17 & 0.0241 & 15.40 & 6.07 & 0.2161 \\
\hline$\geq 3$ & 14.51 & 5.53 & & 14.67 & 4.74 & & 14.50 & 5.60 & \\
\hline \multicolumn{10}{|l|}{ Workplace } \\
\hline Urban & 15.01 & 6.10 & 0.9681 & 18.40 & 12.72 & 0.7166 & 14.83 & 5.63 & 0.9661 \\
\hline Rural & 15.18 & 6.21 & & 18.14 & 6.59 & & 14.99 & 6.17 & \\
\hline Isolated area & 14.89 & 2.32 & & 17.00 & 0.00 & & 14.63 & 2.33 & \\
\hline \multicolumn{10}{|l|}{ Work hours per week } \\
\hline$\leq 45(\mathrm{Q} 1)$ & 13.85 & 4.24 & 0.0335 & 23.00 & 7.00 & 0.2094 & 13.11 & 3.03 & 0.0218 \\
\hline$\leq 50(\mathrm{Q} 2)$ & 14.47 & 4.50 & & 18.00 & 0.00 & & 14.40 & 4.52 & \\
\hline$\leq 56(\mathrm{Q})$ & 14.89 & 5.93 & & 13.20 & 2.77 & & 15.00 & 6.08 & \\
\hline$\leq 57(\mathrm{Q} 4)$ & 16.89 & 8.03 & & 20.75 & 13.67 & & 16.59 & 7.56 & \\
\hline \multicolumn{10}{|l|}{ Stress from immediate superior } \\
\hline High & 14.07 & 6.50 & 0.0003 & 19.00 & 10.14 & 0.7678 & 15.90 & 6.25 & 0.0003 \\
\hline Low & 13.32 & 4.63 & & 16.80 & 6.61 & & 13.09 & 4.43 & \\
\hline \multicolumn{10}{|l|}{ Physical activity (days of activity per week) } \\
\hline 0 per week & 15.47 & 6.35 & 0.0045 & 16.25 & 7.85 & 0.7319 & 15.43 & 6.33 & 0.0067 \\
\hline 1-2 per week & 13.31 & 3.27 & & 16.33 & 2.89 & & 13.17 & 3.23 & \\
\hline$\geq 3$ per week & 16.21 & 7.14 & & 20.33 & 11.45 & & 15.88 & 6.70 & \\
\hline Weight fluctuation & & & & & & & & & \\
\hline Yes (loss or gain in weight $(\geq 3 \mathrm{~kg})$ ) & 15.42 & 6.62 & 0.251 & 19.50 & 10.20 & 0.5549 & 15.17 & 6.32 & 0.3478 \\
\hline None & 14.56 & 4.93 & & 16.00 & 6.00 & & 14.47 & 4.89 & \\
\hline Total & 15.09 & 5.45 & & 18.15 & 8.71 & & 14.91 & 5.82 & \\
\hline
\end{tabular}

\section{DISCUSSION}

Previous studies have assessed how the experience of sexual harassment affects mental health. Sexual harassment is adversely associated with mental health, most notably in PTSD, anxiety, depression and substance use disorders. ${ }^{10} 11{ }^{13} 24$ Our results corroborate those findings. 
Table 3 Regression model coefficients and $p$ values of K-10 Index values

\begin{tabular}{|c|c|c|c|}
\hline & \multicolumn{3}{|l|}{$\mathrm{K}-10$} \\
\hline & $\boldsymbol{\beta}$ & SE & p Value \\
\hline \multicolumn{4}{|l|}{ Experience of sexual harassment } \\
\hline Yes & 3.486 & 1.684 & 0.0397 \\
\hline No & Ref. & - & - \\
\hline \multicolumn{4}{|l|}{ Age (years) } \\
\hline $19-24$ & -0.124 & 1.024 & 0.9034 \\
\hline $25-43$ & Ref. & - & - \\
\hline \multicolumn{4}{|l|}{ Education } \\
\hline$\leq$ High school & 0.425 & 0.996 & 0.6697 \\
\hline$\geq$ Graduate from university & Ref. & - & - \\
\hline \multicolumn{4}{|l|}{ Marital status } \\
\hline Married & Ref. & - & - \\
\hline Unmarried & 3.401 & 1.600 & 0.0348 \\
\hline \multicolumn{4}{|l|}{ Dependent children } \\
\hline Yes & Ref. & - & - \\
\hline No & -1.531 & 1.811 & 0.399 \\
\hline \multicolumn{4}{|l|}{ Military type } \\
\hline Army & Ref. & - & - \\
\hline Navy, Marine & 2.521 & 1.317 & 0.057 \\
\hline Air force & -0.941 & 1.148 & 0.4131 \\
\hline \multicolumn{4}{|l|}{ Branch } \\
\hline Combat branch & -0.408 & 1.239 & 0.7421 \\
\hline Technical service branch & -0.851 & 1.227 & 0.4891 \\
\hline Administrative support branch & 1.439 & 1.528 & 0.3472 \\
\hline Special branch & Ref. & - & - \\
\hline \multicolumn{4}{|l|}{ Rank } \\
\hline Officer & 0.169 & 1.017 & 0.8682 \\
\hline Non-commissioned officer & Ref. & - & - \\
\hline \multicolumn{4}{|l|}{ Service classification } \\
\hline Long-term military service & 0.820 & 1.073 & 0.4454 \\
\hline Short-term military service & 2.067 & 1.052 & 0.0507 \\
\hline Extended military service & Ref. & - & - \\
\hline \multicolumn{4}{|l|}{ Length of services (years) } \\
\hline$\leq 2$ & -0.368 & 1.029 & 0.7208 \\
\hline$\geq 3$ & Ref. & - & - \\
\hline \multicolumn{4}{|l|}{ Workplace } \\
\hline Urban & 0.681 & 2.109 & 0.7472 \\
\hline Rural & 0.932 & 2.049 & 0.6495 \\
\hline Isolated area & Ref. & - & - \\
\hline \multicolumn{4}{|l|}{ Work hours per week } \\
\hline$\leq 45(\mathrm{Q} 1)$ & -2.472 & 1.269 & 0.0528 \\
\hline$\leq 50(\mathrm{Q} 2)$ & -2.119 & 1.206 & 0.0803 \\
\hline$\leq 56(\mathrm{Q} 3)$ & -1.974 & 1.039 & 0.0589 \\
\hline$\leq 57(\mathrm{Q} 4)$ & Ref. & - & - \\
\hline \multicolumn{4}{|l|}{ Stress from immediate superior } \\
\hline High & 2.592 & 0.810 & 0.0016 \\
\hline Low & Ref. & - & - \\
\hline \multicolumn{4}{|l|}{ Physical activity (days of activity per week) } \\
\hline 0 per week & Ref. & - & - \\
\hline 1-2 per week & -1.038 & 0.932 & 0.2664 \\
\hline$\geq 3$ per week & -3.013 & 1.011 & 0.0032 \\
\hline \multicolumn{4}{|l|}{ Weight fluctuation } \\
\hline Yes (loss or gain in weight $(\geq 3 \mathrm{~kg})$ ) & 0.327 & 0.821 & 0.6911 \\
\hline None & Ref. & - & - \\
\hline
\end{tabular}

Table 4 Coefficients and $p$ values from multiple linear regression analysis* of K-10 scores when female military personnel experienced sexual harassment according to marital status, rank, service classification and length of service

\begin{tabular}{|c|c|c|c|c|}
\hline & & \multicolumn{3}{|l|}{ K-10 } \\
\hline & & $\boldsymbol{\beta}$ & SE & p Value \\
\hline \multirow[t]{2}{*}{ Marital status } & Married & -2.500 & 1.574 & 0.1187 \\
\hline & Unmarried & 6.761 & 3.191 & 0.0355 \\
\hline \multirow[t]{2}{*}{ Rank } & Officer & 10.878 & 7.128 & 0.1320 \\
\hline & $\begin{array}{l}\text { Non-commissioned } \\
\text { officer }\end{array}$ & 3.176 & 1.975 & 0.1098 \\
\hline \multirow[t]{3}{*}{ Service classification } & $\begin{array}{l}\text { Long-term military } \\
\text { service }\end{array}$ & 0.668 & 1.989 & 0.7378 \\
\hline & $\begin{array}{l}\text { Short-term military } \\
\text { service }\end{array}$ & 12.014 & 5.342 & 0.0269 \\
\hline & $\begin{array}{l}\text { Extended military } \\
\text { service }\end{array}$ & 4.666 & 2.209 & 0.0385 \\
\hline \multirow[t]{2}{*}{ Length of service (years) } & $\leq 2$ & 11.067 & 4.512 & 0.0159 \\
\hline & $\geq 3$ & 0.398 & 1.906 & 0.8350 \\
\hline
\end{tabular}

*Adjusted for age, education, marital status, dependent children, military type, branch, rank, service classification, length of service, workplace, work hours per week, stress from immediate superior, physical activities and weight fluctuation. K-10, Kessler Psychological Distress Scale 10.

In the present study, we analysed how an experience of sexual harassment impacts the mental health of female members of the ROK Armed Forces. In order to clarify the effects on mental health, the experience of sexual harassment was explored as a socioeconomic element and then analysed with respect to mental health. Generally, K-10 scores were higher for female military personnel who were sexually harassed. Our results verify previous research that shows sexual harassment is associated with emotional problems. ${ }^{25} 26$

This study, unlike others, focused on the unique features of the military. Unmarried female military personnel who were sexually harassed during their military services demonstrated higher K-10 scores than married female military personnel who experienced sexual harassment. Previous research has shown that married people generally exhibit better mental health than unmarried (including never-married and formerly married) persons. $^{27} 28$ The experience of sexual harassment can involve many subjective factors: Korean citizens are relatively private in nature and prefer not to discuss their sexual histories. Korean singles are particularly reluctant to talk openly about sexual intercourse. These properties had a further negative impact on mental health when unmarried female personnel experienced sexual harassment.

The impact on mental health as a result of sexual harassment largely depended upon service classification. Female military personnel with short-term military service or extended military service who experienced sexual harassment showed a more negative impact on their mental health than female military personnel with long-term service. A previous study showed that compared with permanent employees, temporary employees exhibit a higher combined risk estimate for psychological distress. $^{25}$ As there is no obligatory military service for women in Korea, most are selected to join the military as professional soldiers and generally desire long-term service. Women in shortterm service and extended service must be selected in order to continue serving in long term; therefore, they are in the most 
vulnerable military positions. Also, they must receive good reviews and recommendations from immediate superiors in order to be selected for long-term military service. In this situation, some female military personnel are unable to report sexual harassment from their immediate superiors. As shortterm military service personnel are not yet selected into extended military service, they are more vulnerable than extended military service personnel.

Meanwhile, in the $<2$ years of services group, K-10 scores increased when sexual harassment occurred. A previous study showed that new staff had a higher prevalence of psychiatric problems in the workplace as a result of difficulty adapting to the new environment. ${ }^{29}$ When enlisting as younger women, most of the female military personnel demonstrated difficulties with adapting to military service. The experience of sexual harassment only worsens their mental health. Female military personnel in their early $20 \mathrm{~s}$ in the $<2$ years of service group demonstrate additional sensitivity to sexual harassment.

Based on our results, ROK Armed Forces should enforce sexual harassment policies. Emphasis should be placed on the prevention of sexual harassment and not just upon correction and punishment after the incident is reported. The ROK Armed Forces should introduce a sexual harassment questionnaire in order to stay informed of the issue of sexual harassment. The US military has developed a questionnaire that indicates the prevalence of sexual harassment within its ranks. ${ }^{30}$ Second, the ROK military should strengthen gender perspective education. A previous study showed that the biggest reason sexual harassment occurred was the perception by male superiors that female workers were not considered 'professional coworkers' but simply 'women'. 31 The ROK Armed Forces should create an environment that encourages conversation about the differences between male and female soldiers. Third, for the long-term military service selection among the basic female military personnel, it is necessary to develop a selection standard in order to better reflect overall assessment. Also, for fair assessment, specific evidence of the superior's assessment should be submitted. Verification procedures should be developed.

This study is distinguished from others because the results were derived in detail to discriminate personal and ROK Armed Forces characteristics. The study was based on political and cultural characteristics unique to Korea and focused on the relationship between mental health and sexual harassment.

This study should be considered in the context of its strengths and limitations. First, all the data were statistically collected from a survey of ROK Armed Forces conducted by the School of Military Medicine, which is an affiliated organisation of the National Defence. The MHS results represent 600000 ROK Armed Forces. Second, as far as we know, this is the first study to use the MHS data in an attempt to investigate mental health and sexual harassment. The report is based on female military personnel's K-10 scores, which is an excellent screening tool for mental health disorders. ${ }^{32}$ Fourth, this study analysed female military personnel's military characteristics and working environments, in addition to their mental health. Globally, our study supports evidence of the impact of sexual harassment on mental health. In addition, this study provides results that can potentially be used to develop policies to address sexual harassment.

Our study has several limitations. First, because it was crosssectional, it was not possible to identify a causal relationship between the experience of sexual harassment and mental health. Second, male military personnel also experience sexual harassment, and this issue was not explored. In addition, previous studies showed a detrimental effect on the mental health of men when they were victims of sexual harassment. ${ }^{33}$ However, in the 2014 MHS, we could not find any reports of sexual harassment among male military personnel. Third, because of limited access to data, we did not review the clinical records of military personnel with mental health problems. Therefore, we were unable to identify which military personnel had been diagnosed with mental disorders. Fourth, we could not identify the length of being sexually harassed and its impact on functioning within the workplace. Lastly, there was no available information about the offender of sexual harassment.

Based on this research, in order to protect the most vulnerable within the military, it is essential for the military to enforce the sexual harassment prevention policies. A previous study showed that receiving medical care decreased a woman's risk of further sexual assault. ${ }^{34}$ Following sexual harassment, psychiatric treatment and ongoing management are integral to prevent worsening of mental health. Future studies should focus on the effects of sexual harassment on mental health based on the identity of the offender and the impact of psychiatric treatment after the experience of sexual harassment, as well as an association with the length of being sexually harassed and mental health and the impact of sexual harassment on functioning within the workplace. New findings would have important implications for sexual harassment policymaking.

\section{CONCLUSION}

We have confirmed that there is a differentiable relationship between the experience of sexual harassment and mental health. We also verified that the relationship was impacted by female military personnel's service classification and length of service. The results provide helpful information to minimise negative psychological effects after sexual harassment and to promote sexual harassment prevention policy.

\section{Collaborators TKK, H-CL, SGL, K-TH and E-CP.}

Contributors TKK has received commissioned education at Yonsei University, Seoul, Republic of Korea, designed the study, researched the data, performed statistical analyses and wrote the manuscript. H-CL, SGL, K-TH and E-CP contributed to the discussion and reviewed and edited the manuscript. E-CP is the guarantor of this work and as such, takes responsibility for the integrity of the data and the accuracy of the data analysis. The English in this article has been checked by Amanda Hendrix from BioScience Writers (https://www.biosciencewriters.com/ EditingCertificateVerify.aspx / Validation Code:

2501A2ED-0D9E-44AE-B008-D0666002A645).

\section{Competing interests None declared.}

Ethics approval The Institutional Review Board (IRB) of the Armed Forces Medical Command (AFMC) provided formal ethics approval for the MHS data sets (IRB approval number AFMC-14-IRB-004).

Provenance and peer review Not commissioned; externally peer reviewed.

Open Access This is an Open Access article distributed in accordance with the Creative Commons Attribution Non Commercial (CC BY-NC 4.0) license, which permits others to distribute, remix, adapt, build upon this work non-commercially, and license their derivative works on different terms, provided the original work is properly cited and the use is non-commercial. See: http://creativecommons.org/ licenses/by-nc/4.0/

\section{REFERENCES}

1 Doe SS. Cultural factors in child maltreatment and domestic violence in Korea. Child Youth Serv Rev 2000;22:231-6

2 Choi M, Harwood J. A hypothesized model of Korean women's responses to abuse. J Transcult Nurs 2004;15:207-16.

3 Hong W, Yamamoto J, Chang DS, et al. Sex in a Confucian society. J Am Acad Psychoanal 1993;21:405-19.

4 Ministry of National Defense RoK. 2014 Defense white paper. Seoul: Ministry of National Defense, 2014. http://www.mnd.go.kr/user/mnd/upload/pblictn/ PBLICTNEBOOK_201506120206080360.pdf; last accessed Feb 17, 2016 
5 Ministry of National Defense, Republic of Korea. The last 5 years of sexual harassment or sexual abuse offenders disciplinary Status, Report of parliamentary inspection of the administration 2014. Seoul: Ministry of National Defense, 2014.

6 Organization for Economic Cooperation Development Staff. Health at a glance 2013: OECD Indicators: OECD, 2013. (accessed 17 Feb 2016).

7 Jang SI, Nam JM, Choi J, et al. Disease management index of potential years of life lost as a tool for setting priorities in national disease control using OECD health data. Health Policy 2014;115:92-9.

8 Ministry of National Defense, Republic of Korea. Prevalence of depression in the military 2012. Seoul: Ministry of National Defense, 2013.

9 Antecol H, Cobb-Clark D. The sexual harassment of female active-duty personnel: effects on job satisfaction and intentions to remain in the military. J Econ Behav Organ 2006;61:55-80.

10 Hankin CS, Skinner KM, Sullivan LM, et al. Prevalence of depressive and alcohol abuse symptoms among women VA outpatients who report experiencing sexual assault while in the military. J Trauma Stress 1999;12: 601-12.

11 Yaeger D, Himmelfarb N, Cammack A, et al. DSM-IV diagnosed posttraumatic stress disorder in women veterans with and without military sexual trauma. J Gen Intern Med 2006;21(Suppl 3):S65-9.

12 Gruber JE, Fineran S. The impact of bullying and sexual harassment on middle and high school girls. Violence Against Women 2007;13:627-43.

13 Kimerling R, Street AE, Pavao J, et al. Military-related sexual trauma among Veterans Health Administration patients returning from Afghanistan and Iraq. Am J Public Health 2010;100:1409.

14 Bebbington PE, Cooper C, Minot S, et al. Suicide attempts, gender, and sexual abuse: data from the 2000 British Psychiatric Morbidity Survey. Am J Psychiatry 2009;166:1135-40

15 Cobb Scott J, Pietrzak RH, Southwick SM, et al. Military sexual trauma interacts with combat exposure to increase risk for posttraumatic stress symptomatology in female Iraq and Afghanistan veterans. J Clin Psychiatry 2014;75:637-43.

16 Brewin CR, Garnett R, Andrews B. Trauma, identity and mental health in UK military veterans. Psychol Med 2011;41:1733-40.

17 Luterek JA, Bittinger JN, Simpson TL. Posttraumatic sequelae associated with military sexual trauma in female veterans enrolled in VA outpatient mental health clinics. J Trauma Dissociation 2011;12:261-74

18 Spies G, Stein D, Roos A, et al. Validity of the Kessler 10 (K-10) in detecting DSM-IV defined mood and anxiety disorders among pregnant women. Arch Womens Ment Health 2009;12:69-74.
19 Slade T, Grove R, Burgess P. Kessler psychological distress scale: normative data from the 2007 Australian national survey of mental health and wellbeing. Aust N Z J Psychiatry 2011;45:308-16.

20 Cornelius BL, Groothoff JW, van der Klink JJ, et al. The performance of the K10, K6 and GHQ-12 to screen for present state DSM-IV disorders among disability claimants. BMC Public Health 2013:13:128.

21 Kim YS. Validation of the Korean versions of K-10 and K6 among low-income individuals. Ment Health Soc Work 2011;37:65-88.

22 Browne MAO, Wells JE, Scott KM, et al. The Kessler psychological distress scale in Te Rau Hinengaro: the New Zealand mental health survey. Aust N Z J Psychiatry 2010:44:314-22

23 Till FJ. Sexual Harassment. A Report on the Sexual Harassment of Students. 1980. http://files.eric.ed.gov/fulltext/ED197242.pdf. (accessed 17 Feb 2016).

24 Kelly UA, Skelton K, Patel M, et al. More than military sexual trauma: interpersonal violence, PTSD, and mental health in women veterans. Res Nurs Health 2011;34:457-67.

25 Virtanen M, Kivimäki M, Joensuu M, et al. Temporary employment and health: a review. Int J Epidemiol 2005;34:610-22.

26 Kimerling R, Gima K, Smith MW, et al. The Veterans Health Administration and military sexual trauma. Am J Public Health 2007;97:2160-6.

27 Afifi TO, Cox BJ, Enns MW. Mental health profiles among married, never-married, and separated/divorced mothers in a nationally representative sample. Soc Psychiatry Psychiatr Epidemiol 2006;41:122-9.

28 Holt-Lunstad J, Birmingham W, Jones BQ. Is there something unique about marriage? The relative impact of marital status, relationship quality, and network social support on ambulatory blood pressure and mental health. Ann Behav Med 2008:35:239-44.

29 Wong DFK, He X, Leung G, et al. Mental health of migrant workers in China: prevalence and correlates. Soc Psychiatry Psychiatr Epidemiol 2008:43:483-9.

30 Street AE, Stafford J, Mahan CM, et al. Sexual harassment and assault experienced by reservists during military service: prevalence and health correlates. J Rehabil Res Dev 2008;45:409.

31 McDonald P. Workplace sexual harassment 30 years on: a review of the literature Int J Manag Reviews 2012;14:1-17.

32 Kessler RC, Barker PR, Colpe L, et al. Screening for serious mental illness in the general population. Arch Gen Psychiatry 2003;60:184-9.

33 Street AE, Gradus JL, Stafford J, et al. Gender differences in experiences of sexual harassment: data from a male-dominated environment. J Consult Clin Psychol 2007;75:464

34 McFarlane J, Malecha A, Watson K, et al. Intimate partner sexual assault against women: frequency, health consequences, and treatment outcomes. Obstet Gynecol 2005:105:99-108. 